\title{
Les Occlusions Intestinales Par Nœud Ileo-Sigmoïdien : Aspects Diagnostiques, Thérapeutiques Et Pronostiques
}

\author{
James Didier. L \\ Département de chirurgie, Hôpital National de Niamey
}

Chaibou MS

Département d'anesthésie et de réanimation, Hôpital National de Niamey

Adama. S

Idé $K$

Abdoulaye MB

Département de chirurgie, Hôpital National de Niamey

Daddy. $\boldsymbol{H}$

Département d'anesthésie et de réanimation, Hôpital National de Niamey

Adakal. $O$

Faculté des Sciences de la Santé de l'Université de Maradi /Niger

Adamou $\boldsymbol{H}$

Département de chirurgie, Hôpital National de Zinder

Marouf MI

Maternité Issaka Gazoby

Sidibe. $T$

Service de Radiologie, Hôpital national de Niamey, Niger

Sani. $R$

Département de chirurgie, Hôpital National de Niamey

Doi: 10.19044/esj.2017.v13n33p254 URL:http://dx.doi.org/10.19044/esj.2017.v13n33p254

\begin{abstract}
Introduction: Ileosigmoid knot (ISK), also known as compound volvulus or double volvulus, is a rare disease and affects mostly male subjects in their fourth decade. Delayed diagnosis often leads to complications with a high incidence of digestive necrosis. Materials and methods: This was a 10-year retrospective, descriptive and analytical study from 1 January 2007 to 31 December 2016, which covered all patients operated emergently for ISK in the surgical and surgical specialty departments at the Niamey National Hospital (NNH). Results: The series involved 8 cases of ISK, representing $0.82 \%$ of intestinal obstructions. There
\end{abstract}


were 7 men and 1 woman. The average age of patients was 44.33 years with extremes ranging from 25 to 60 years. The main clinical signs were: abdominal pain (100\%), inability to pass gas and stool $(100 \%)$, vomiting $(87.5 \%)$ and abdominal meteorism $(87.5 \%)$. Four patients $(50 \%)$ had a clear obstructive syndrome and the other 4 patients (50\%) had associated signs of peritoneal irritation. Paraclinical diagnosis was difficult because of atypical clinico-radiological signs, but in all cases an abdominal x-ray was performed and showed a double loop of dilated sigmoid shadow in $87.5 \%$ of the cases. Laparotomy was the first approach used in all patients and led to a $50 \%$ necrosis rate. Hartmann's colectomy and ideal colectomy were used in identical proportions, with $50 \%$ each. Ideal colectomy was characterized by a morbidity rate of $50 \%$ and a mortality rate of $25 \%$ compared to $0 \%$ for Hartmann's procedure. The overall morbidity was $25 \%$ and the overall mortality was $12.5 \%$. The average hospital stay was 65.87 days (range 17 to 128 days). The average time before reestablishment of digestive continuity was 50.37 days (range 31-128 days). Conclusion: Ileosigmoid knot is a rare condition at Niamey national hospital where the diagnostic approach is similar to that of occlusions in general, but remains difficult due to its uncommonness and atypical clinico-radiological signs. Necrosis rates and postoperative complications remain high.

Keywords: Ileosigmoid knot, intestinal obstruction, volvulus, colectomy, Niger

\section{Résumé}

Introduction: Le nœud ileo-sigmoïdien (NIS) est une pathologie rare et touche majoritairement les sujets de sexe masculin de la quatrième décade. Le retard diagnostic conduit souvent à des complications avec pour corollaire une incidence élevée de nécrose digestive. Matériels et méthodes : Il s'agissait d'une étude rétrospective, descriptive et analytique sur 10 ans allant du $1^{\text {èr }} \quad$ janvier 2007 au 31 décembre 2016 et qui a concerné tous les patients opérés en urgence pour NIS dans les services de chirurgie et spécialités chirurgicales de l'hôpital national de Niamey (HNN). Résultats : La série a concerné 8 cas de NIS soit $0,82 \%$ des occlusions intestinales. Il s'agissait de 7 hommes et une femme. L'âge moyen des patients était de 44,33 ans avec des extrêmes allant de 25 à 60 ans. Les principaux signes cliniques sont représentés par la douleur abdominale (100\%), l'arrêt des matières et gaz $(100 \%)$, les vomissements $(87,5 \%)$ et le météorisme abdominal (87,5\%). Quatre patients (50\%) présentaient un tableau de syndrome occlusif franc et les 4 autres patients $(50 \%)$ présentaient un syndrome d'irritation péritonéale associée. Le diagnostic paraclinique était difficile du fait d'une atypie clinico- radiologique mais dans tous les cas un 
ASP a été réalisé et montrait une image en arceau à double jambage dans $87,5 \%$ des cas. La laparotomie était la voie d'abord utilisée chez tous les patients et avait permis de découvrir un taux de nécrose de $50 \%$. La colectomie selon Hartmann et la colectomie idéale ont été utilisées dans des proportions identiques soit $50 \%$ chacune. La colectomie idéale est marquée par un taux de morbidité de $50 \%$ et un taux de mortalité de $25 \%$ contre $0 \%$ pour la colectomie selon Hartmann. La morbidité globale était de $25 \%$ et la mortalité globale était de 12,5\%. Le séjour hospitalier moyen était de 65,87 jours (extrêmes de 17 à 128 jours). Le délai moyen de rétablissement de la continuité digestive était de 50,37 jours (extrêmes de 31 à 128 jours). Conclusion: le Le nœud ileo-sigmoïdien est une affection rare à l'hôpital national de Niamey où la démarche diagnostique rejoint celle des occlusions en général mais reste difficile, en raison de sa rareté et d'une atypie clinicoradiologique. Le taux de nécrose et les complications postopératoires restent élevés.

Mots-clés : Nœuds iléo-sigmoïdien, occlusions, volvulus, colectomie, Niger

\section{Introduction}

Le nœud iléo-sigmoïdien (NIS) ou double volvulus iléo-sigmoïdien est un enroulement du grêle autour de la base du colon sigmoïde, réalisant ainsi une occlusion intestinale par strangulation bifocale du sigmoïde et de l'iléon (Arthur, 2007). En fonction de la force de serrage du nœud et de l'engagement du mésentère, cette strangulation peut causer rapidement la nécrose ischémique de l'iléon et du colon (74-80\%). L'évolution se fait dans 56\% des cas vers un état de choc hypovolémique (Alver, 2005 Thambi, 1988 Johnson, 1986 Burrah, 2010). Le NIS est une pathologie rare, décrite dans les régions d'Afrique, d'Asie représentant 7,6\% de l'ensemble des volvulus du sigmoïde, mais encore plus rare en Europe (en France où il est, plus vraisemblablement, moins de 1\%) (Atamanalp, 2004 Alver, 1993). Le retard au diagnostic conduit souvent à des complications avec pour corollaire une incidence élevée de nécrose digestive, variant entre $74 \%$ et $100 \%$ (Atamanalp, 2004 Raveenthiran, 2001). Le nœud iléo-sigmoïdien est considéré comme une vraie urgence chirurgicale qui évolue rapidement vers la nécrose intestinale. Ainsi La connaissance du mécanisme de cette pathologie est essentielle afin de pouvoir poser précocement le diagnostic pour permettre une prise en charge chirurgicale rapide (Thambi, 1988). Le diagnostic repose le couple cliche abdominale sans préparation (avec une sensibilité autour de 60\%) et/ou après lavement opaque (avec une sensibilité très améliorée $80 \%$ et spécificité correcte) (Thambi, 1988). Mais de nos jours le scanner représente l'élément clé du diagnostic du NIS, il permettrait la mise en évidence d'un syndrome obstructif et de son mécanisme avec une 
bonne spécificité ( $>90 \%)$ et une bonne sensibilité ( $>95 \%)$ (Vandendries, 2007). Son traitement est essentiellement chirurgical et La mortalité est élevée, variant souvent entre 15\% et 30\% (Vandendries, 2007 Kedir, 1998). Le pronostic est sévère, la mortalité est de $6,8 \%$ à $8 \%$ dans les formes non gangréneuse et $20 \%$ à $100 \%$ dans les formes gangréneuses.

\section{Matériels et méthodes}

Il s'agissait d'une étude rétrospective, descriptive et analytique sur 10 ans allant du 1èr janvier 2007 au 31 décembre 2016. Étaient inclus dans l'étude, Les patients opérés pour occlusion par nœud iléo-sigmoïdien. N'étaient pas incluent, les patients opérés pour autres causes d'occlusions.

\section{Résultats}

Durant la période d'étude, 970 cas d'occlusions intestinales ont été colligés parmi lesquelles 8 cas d'occlusion intestinale par nœud iléosigmoïdien(NIS). Le NIS représentait $0,82 \%$ (soit 0,8 cas par an) des occlusions intestinales, $4,76 \%$ des volvulus du colon sigmoïde (VCS) et $0,12 \%$ des urgences abdominales. Le sexe masculin représentait $87,5 \%$ des cas. L'âge moyen des patients était de 44,3 ans avec des extrêmes compris entre 25 et 60 ans. . Le délai moyen d'admission est de 60 heures soit 2,5 jours et les patients admis entre 1 et 3 jours du début des signes représentaient $75 \%$ des cas. La déshydratation était retrouvée dans $50 \%$ des cas. L'état général à l'admission était altéré dans $12,5 \%$ des cas, lié surtout à la fièvre et à la déshydratation. La douleur abdominale et l'arrêt des matières et gaz étaient retrouvés chez tous les patients. Le Météorisme était retrouvé chez 7 patients soit $87,5 \%$ des cas. Le syndrome d'irritation péritonéal était associé dans $50 \%$ des cas. La radiographie d'abdomen sans préparation (ASP) était demandée pour tous les patients. L'image en arceau à double jambage était retrouvée dans $87,5 \%$ des cas et des niveaux hydro-aériques mixtes (NHA) dans $50 \%$ (voir tableau I).

Tableau I : Répartition des patients selon les types d'images radiologiques

\begin{tabular}{cccc}
\hline & Effectifs & Pourcentage & $(\%)$ \\
Image sur l'ASP & & 87,5 \\
Image en arceau & 7 & 50 \\
NHA mixtes & 4 & \\
\hline
\end{tabular}




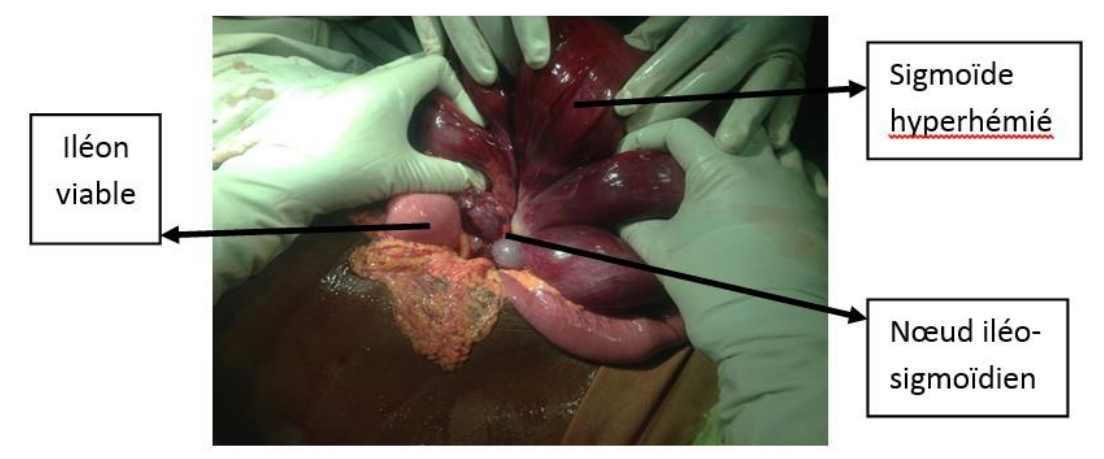

Image 1: nœud ileosigmoidien sans nécrose : image montrant le nœud

Tous les patients de l'étude ont bénéficié d'une réanimation préopératoire ayant consisté à une réhydratation à base de cristalloïde, la pose d'une sonde naso-gastrique et d'une sonde urinaire, d'une antibiothérapie et des antalgiques. La totalité des patients étaient opérés sous anesthésie générale et la voie d'abord a été une laparotomie médiane à cheval sur ombilicale. Les liquides séro-hématique et pyostercoral étaient retrouvés dans des proportions égales de $50 \%$. La perforation était retrouvée dans $50 \%$ des cas. Il s'agit toutes des perforations sigmoïdienne diastasiques par contre la nécrose était uniquement survenue sur le grêle dans $50 \%(\mathrm{n}=4)$ (voir tableau II).

Tableau II : Répartition des patients selon l'état de l'anse intestinale.

\begin{tabular}{cccccc}
\hline $\begin{array}{c}\text { Etat de } \\
\text { l'anse }\end{array}$ & Perforation & $\begin{array}{c}\text { Pas } \\
\text { de perforation }\end{array}$ & Nécrose & $\begin{array}{c}\text { Pas } \\
\text { de nécrose }\end{array}$ & $\begin{array}{c}\text { Perforation et } \\
\text { nécrose associées }\end{array}$ \\
\hline Colon & 4 & 4 & 0 & 8 & 0 \\
Grêle & 0 & 8 & 4 & 4 & 0 \\
\hline
\end{tabular}

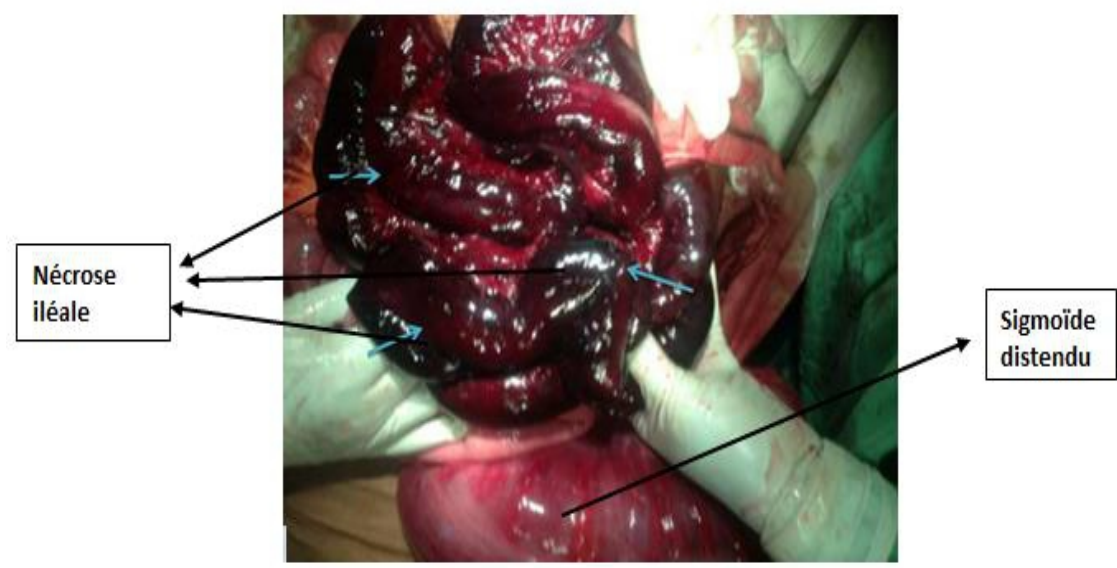

Image 2 : Nœud iléo-sigmoïdien avec nécrose étendue de l'intestin grêle Côlon sigmoïde distendu mais de bonne viabilité (flèches). 
La colectomie selon Hartmann était réalisée chez 50\% des patients. La dévolution suivie de résection anastomose immédiate (colectomie idéale) du colon a concerné 4 patients soit $50 \%$. Pour les 4 cas de nécrose du grêle, il a été réalisé une résection anastomose d'emblée, pour le reste une détorsion simple a été faite. Il n'y avait pas eu de double anastomose colique et grelique (Tableau III).

Tableau III : Répartition des patients selon Les gestes.

\begin{tabular}{cccccc}
\hline Gestes & $\begin{array}{c}\text { Détorsion } \\
\text { iléale sans } \\
\text { résection }\end{array}$ & $\begin{array}{c}\text { Résection iléale } \\
\text { Anastomose } \\
\text { immédiate }\end{array}$ & $\begin{array}{c}\text { Colectomie } \\
\text { idéale }\end{array}$ & $\begin{array}{c}\text { Colectomie } \\
\text { selon Hartmann }\end{array}$ & total \\
\hline Colon & 0 & 0 & 4 & 4 & 8 \\
Grêle & 4 & 4 & 0 & 0 & 8 \\
\hline
\end{tabular}

Les complications étaient de $25 \%(n=2)$. Ils s'agissaient de suppurations pariétales associées à une éviscération fixée. La mortalité est de $12,5 \%(n=1)$ après une colectomie idéale. Mais le décès n'était pas survenu en post opératoire immédiat. La cause n'a pas été clairement élucidée. La durée moyenne de séjour était de 65,87 jours avec des extrêmes allant de 17 à 139 jours. Le rétablissement de la continuité digestive du colon sigmoïde était réalisé dans un délai moyen de 50,37 jours (extrêmes allant de 31 à 128 jours).

\section{Discussions}

$\mathrm{Au}$ cours de notre étude, 970 cas d'occlusions intestinales sont recensés dans les services de chirurgie et spécialités chirurgicales de l'hôpital national de Niamey dont 8 cas du NIS avec une fréquence de $0,82 \%$, soit une incidence de 0,8 cas par an. Ce résultat est proche de ceux de certains auteurs qui ont retrouvé respectivement 0,55 et 0,71 cas/an (Atamanalp, 2004 Puthu, 1991). Cependant d'autres publications en ont rapporté plus avec 3,66 cas/an (Vaez-Zadeh, 1970). Ces résultats montrent que le NIS est une pathologie rare autant dans les pays en voie de développement que dans les pays développés. Dans notre série, l'âge moyen est de 44,33 avec des extrêmes allant de 25 à 60 ans. Ce résultat est proche de ceux retrouvés dans plusieurs séries qui ont retrouvé respectivement un âge moyen de 43 et 45 ans (Raveenthiran, 2001 Akgun, 1997). Par contre d'autres ont trouvé un âge moyen plus jeune est rapporté entre 32 et 38,66 ans (Hachimato, 2004 Toure, 2013). Ceci montre que le NIS est plus fréquent dans la troisième et quatrième décade de la vie Quel que soit les régions du monde. Dans cette série, le sexe masculin prédomine. Cette tendance est retrouvée dans plusieurs autres séries (Toure, 2013 Shephead 1967). Cette prédominance masculine pourrait s'expliquer par le fait que les hommes ont un mésocôlon très haut et un bassin étroit alors que les femmes ont un bassin large et 
une musculature moins tonique que celle des hommes ce qui permet une dévolvulation spontanée. Toutefois, pendant la grossesse, elles deviennent plus exposées avec des graves accidents occlusifs notamment au cours du troisième trimestre, en post partum ou après une intervention sur la filière génitale telle que la césarienne, l'annexectomie (Udezue, 1990).

Nos patients sont admis dans un délai moyen de 2,5 jours avec des extrêmes de 1 à 4 jours. Ce résultat est comparable à ceux retrouvés dans les séries africaines et asiatiques (Shephead, 1967 Raveenthiran, 2001) qui ont rapporté un délai moyen de 2,2 et 3 jours. Par contre dans les pays développés ce délai est court (Hachimato, 2004)]. L'état général des patients est altéré dans un huitième des cas. Ce résultat est diffèrent de ceux de certaines séries où le tiers voir plus de la moitié des patients avaient un état général altéré (Thambi, 1988 Toure, 2013). Dans notre série, tous les patients présentent une douleur abdominale. C'est le principal symptôme, constant des abdomens aigues incluant ainsi les occlusions par nœuds ileosigmoidien comme rapporté dans plusieurs publications (Atamanalp, 2004 Vaez-Zadeh, 1970 Shephead, 1967). Dans notre étude la majorité des patients présentaient des vomissements à l'admission. Ce résultat est similaire à ceux d'alver qui ont retrouvé 86,76\% (Alver, 2005). Cependant dans d'autres séries, tous les patients ont présenté des vomissements (Atamanalp, 2004 Raveenthiran, 2001). L'arrêt des matières et gaz ainsi que le météorisme sont retrouvés chez presque tous les patients. Ces signes sont souvent constants tel que rapporté dans la littérature même si c'est à des proportions variables, avec pour l'arrêt des matières et gaz de $66,7 \%$ à $100 \%$ (Atamanalp, 2004) et le météorisme de 27,27 à 100\% (Raveenthiran, 2001 Shephead, 1967 Kakar, 1981). Le tableau clinique d'un nœud ileosigmoidien est celui d'une occlusion par strangulation avec des signes mixtes, ceux d'une occlusion haute avec des vomissements précoces ainsi que ceux d'une occlusion basse avec un arrêt de matières et gaz précoces avec un météorisme parfois important.

Dans cette série, la moitié des patients présentent une défense abdominale. Ce résultat n'est pas loin de ceux d'Atamanalp et K. Vaez qui ont respectivement rapporté 50 et 55,6\% des cas ((Atamanalp, 2004 VaezZadeh, 1970). Ceci traduit le plus souvent une complication évolutive du NIS vers celui d'une péritonite secondaire (soit à une perforation diastatiques où celle d'une nécrose ischémique). Celle-ci est gravissime car stercorale et ischémique. L'abdomen sans préparation est demandé chez tous les patients et a retrouvé une image en arceau à double jambage avait dans la majorité des cas. Ce résultat est de peu moins que celui de Toure. Ao qui a trouvé cette image en arceau à double jambage chez tous leurs patients (Toure, 2013). Les niveaux hydro-aériques mixtes sont retrouvés dans la moitié des cas pouvant signifier soit une aggravation de l'occlusion ou une péritonite. 
Dans notre étude, tous les patients n'ont pas bénéficié de scanner, pour asseoir le diagnostic de NIS en préopératoire. Ceci note la limite des moyens techniques de nos systèmes de santé d'une part et d'autre part l'insuffisance pécuniaire des patients.. Cependant dans la littérature, l'examen scannographique est un examen clé et primordiale permettant de suspecter le diagnostic en mettant en évidence une dilatation du sigmoïde et des anses grêles le plus souvent en état de souffrance associées à un défaut de rehaussement pariétal (Sani, 2011). Dans cette série tous les patients ont réalisé le bilan préopératoire. Ce résultat était similaire aux données de la littérature selon lesquelles tout patient doit bénéficier d'un bilan préopératoire d'opérabilité minimum même en urgence (Atamanalp, 2004 Toure, 2013 Sani, 2011).

Avant toute chirurgie le traitement médical et la réanimation soulagent le patient, corrigent et préviennent les troubles hydroélectrolytiques en pré, per et postopératoire. Ce qui est le cas pour les patients de cette série. Pour Le traitement chirurgical, la voie d'abord a été une laparotomie médiane sus et sous ombilicale. L'épanchement intrapéritonéal est dans la moitié des cas sérohématique et dans l'autre moitié, stercopurulent secondaire à une perforation diastatique du sigmoïde. La nécrose est retrouvée dans $50 \%$ également. Ce taux est inférieur à ceux d'autres séries qui ont retrouvé de nécrose dans tous les cas (Vaez-Zadeh, 1970 Toure, 2013). Dans notre étude la nécrose est iléale uniquement présente chez un huitième des patients admis entre un et deux jours et dans plus d'un tiers des patients admis entre deux à trois jours. Notre résultat concorde avec ceux de la littérature selon lesquels la survenue de la nécrose intestinale n'est pas liée seulement à la durée d'évolution de la maladie, mais également au degré de striction de l'anse intestinale. Cependant le long délai d'admission constitue également un facteur aggravant (Alver, 2005 Sani, 2011). On n'a pas trouvé des causes à l' origine du NIS en dehors d'un cas de dolicho-sigmoide. L'étiologie du NIS donne lieu à des controverses. L'hyperlaxité du méso, l'allongement et la proximité des pieds de l'anse sigmoïdienne et aussi la distension de la paroi abdominale antérieure sont les principaux facteurs anatomiques incriminés. Les adhérences post chirurgicales, les hernies internes, le diverticule de Meckel et les malrotations de l'anse intestinale primitive pourraient aussi être des facteurs favorisants (Puthu, 1991).

Dans cette étude, la colectomie selon Hartmann a été réalisée chez la moitié des patients. Ce qui est proche de ceux de certaines séries où cette technique est utilisée dans deux tiers des cas (60 à 66\%) (Atamanalp, 2004 Toure, 2013). Par contre plus que ceux d'autres où celle-ci est réalisée dans moins d'un tiers des cas (Puthu, 1991). La colectomie idéale a été réalisé chez également l'autre moitié des patients, proche de celui de PUTHU en 
Inde qui a rapporté $57,14 \%$ des cas (Puthu, 1991). Cependant des auteurs ont rapporté des cas moindre entre 33 et $38 \%$ [2,15]. Dans notre série, la morbidité globale est de $25 \%$. Ce résultat est proche de celui d'Atamanalp qui a retrouvé $22,37 \%$ des cas (Atamanalp, 2004). Cependant ce taux est inférieur à ceux d'autres études où il varie de 36,36 à 40\% (Vaez-Zadeh, 1970 Eugene, 1993). La mortalité est de 12,5\% ( un patient) proche de celui de certaines séries qui ont retrouvé $11,1 \%$ des cas (Atamanalp, 2004) contrairement à d'autres où le taux est plus élevé variant de 28,7 à $47,8 \%$ (Puthu, 1991 Eugene, 1993 Toure2013). La morbidité dans notre série est dominée par la fistule entero-pariétale et l'éviscération contrairement aux données de la littérature qui sont dominées par la septicémie (Eugene, 1993). Ces complications sont souvent sans gravité mais pourraient prolonger l'hospitalisation et imputer sur l'économie du patient. La mortalité significativement élevée pourrait être expliquée par les nécroses intestinales, la péritonite, le retard de consultation dans la prise en charge dans nos pays mais aussi le mauvais état général des patients. Dans notre série la colectomie idéale est corrélée à un taux de morbidité plus élevé par rapport à la colectomie selon Hartmann et un taux de mortalité également plus important que dans la technique de Hartmann. La grande morbidité dans la colectomie idéale s'expliquerait par les conditions générales de l'intervention (urgence, absence de préparation des patients, mauvais état général des patients, l'état de l'anse intestinale et de la cavité abdominale). Ces constatations concordent avec les données de la littérature (Atamanalp, 2004 Shephead, 1967). Le rétablissement de la continuité digestive dans notre série est réalisé dans un délai moyen de 50,37 jours ( extrêmes allant de 31 à 128 jours ), et la durée moyenne de séjour hospitalier est de 65,87 jours ( extrêmes allant de 17 à 139 jours) moins que celui rapporté par d'autres auteurs qui retrouvaient une durée moyenne de 17,1 jours (Atamanalp, 2004). Ce long séjour hospitalier pourrait s'expliquer par le fait que les patients restent hospitalisés jusqu'au rétablissement de la continuité digestive et la guérison dans nos pratiques mais aussi la survenue des complications en postopératoire dont la prise en charge est longue et couteuse pour le patient.

\section{Conclusion}

Le nœud iléo-sigmoïdien constitue une cause rare d'occlusion intestinale .L'étiologie de cette pathologie reste toujours mal élucidée. La démarche diagnostique rejoint celle des occlusions en général mais reste difficile, en raison de sa rareté et d'une atypie clinico-radiologique. Dans la majorité des cas le diagnostic est porté en peropératoire. La colectomie selon Hartmann doit être privilégiée à la colectomie idéale en raison de sa morbidité et mortalité élevées. Le pronostic dépend essentiellement du délai 
d'admission, de l'état général du patient, de la présence ou non de la nécrose des anses et des circonstances de l'intervention chirurgicale.

\section{References:}

1. Arthur. V, Pascal. A, Hubert. R and al. Le nœud ileosigmoidien: une cause improbable d'occlusion intestinale primitive par strangulation bifocal. Gastroenterol Clin Biol 2007; 31: 975-977.

2. Alver O, Apaydin B, Yigitbasi R, et al. internal herniation concurrent with knotting ileosigmoid or sigmoid volvulus : presentation of 12 patients, surgery $2005 ; 137: 372-7$.

3. Thambi Dorai Cr. Ileosigmoid knot - a case report. Sing med j. 1988; 29:413-415.

4. Johnson Cd. An unusual volvulus - the ileosigmoid knot. Postgraduate Medical Journal. 1986; 62:47-49.

5. Burrah R, Menon A, Pathan H, et al. The ileosigmoid knot. Indian J Surg. 2010; 72:140-142.

6. Atamanalp S.S, Oren D, Yildirgan Mi, et al. Ileosigmoidal Knotting: Outcome in 63 Patients. Dis Colon Rectum. 2004 Jun; 47: 906-10.

7. Alver O, Ören D, Tireli M, et al. Ileosigmoid knotting in Turkey Review of 68 cases. Dis Colon Rectum. 1993 Dec; 36(12):1139-47.

8. Raveenthiran V. The ileosigmoid knot: new observations and changing trends, dis colon rectum 2001; 44: 1196-200.

9. Vandendries. Mc MC. Jullès, I. BoulayColetta et al. Intérêt du scanner multidétecteur à la phase aiguë des volvulus du côlon. Digestif diagnostique et interventionnel. 2007. Paris. Congres 2007, Société française de radiologie 2007.

10. Kedir M, Kotsso B, Messele G. Ileosigmoidd knotting in Gondar teaching hospital north-west Ethiopia. Ethiop Med J 1998; 36:25560.

11. Puthu D, Rajan N, Shenoy Gm, et al. The ileosigmoid knot. Dis colon Rectum 1991 ; 34 : 161-6.

12. K. Vaez-Zadeh, M.D, W. Durz et al. Ileosigmoid Knotting. Annals of Surgery 280:1028, 1970

13. AKGUN Y. Management of Ileosigmoid knotting Br Surg. 1997; 84:672-673

14. Hachimato T, Yamaguchi J, Fujioka $\mathrm{H}$ et al. Two cases of Ileosigmoid knot: the youngest reported patient and CT findings. Hepato-gastroenterology. 2004; 51:771-773.

15. Toure Ao, Ka I, Gnangnon F, et coll. Nœud iléo-sigmoïdien à propos de 3 cas. Livre des résumés. Congres conjoint mondial et panafricain de chir. 2013 p 47. 
16. Shephead J.J. Ninety two cases of Ileosigmoid knotting in Uganda. Br J Surg. 1967; 54:561-566.

17. Udezue No. Sigmoid volvulus in Kaduna, Nigeria. Dis colon rectum 1990;33: 647-9

18. Kakar A, Bahatnagar B.N. Ileosigmoid knotting: a clinical study of 11cases Aust NZJ Surg. 1981, 51: 456-458. Doi: 10.1111/J. 14452197.1981.

19. Sani R, Didier J.L, Chaibou M.S, et al. Urgences coliques chirurgicales de l'adulte à l'hôpital national de Niamey (Niger). J Afr Chir Digest 2011; Vol 11 (1): 1162 - 1166.

20. Eugene J. Gibney F.R, Charles N, et al. Ileosigmoid Knotting. Dis colon Rectum 1993; 36:855-857. 